\title{
Tick-borne encephalitis virus in ticks detached from humans and follow-up of serological and clinical response.
}

Pontus Lindblom, Peter Wilhelmsson, Linda Fryland, Johanna Sjowall, Mats Haglund, Andreas Matussek, Jan Ernerudh, Sirkka Vene, Dag Nyman, Åshild Andreassen, Pia Forsberg and Per-Eric Lindgren

\section{Linköping University Post Print}

\section{Tweet}

N.B.: When citing this work, cite the original article.

Original Publication:

Pontus Lindblom, Peter Wilhelmsson, Linda Fryland, Johanna Sjowall, Mats Haglund, Andreas Matussek, Jan Ernerudh, Sirkka Vene, Dag Nyman, Åshild Andreassen, Pia Forsberg and PerEric Lindgren, Tick-borne encephalitis virus in ticks detached from humans and follow-up of serological and clinical response., 2014, Ticks and Tick Borne Diseases, (5), 1, 21-28.

http://dx.doi.org/10.1016/j.ttbdis.2013.07.009

Copyright: Elsevier

http://www.elsevier.com/

Postprint available at: Linköping University Electronic Press

http://urn.kb.se/resolve?urn=urn:nbn:se:liu:diva-103491 


\section{Tick-borne encephalitis virus in ticks detached from humans and follow-up of serological and clinical response}

Pontus Lindblom, ${ }^{1 *}$ Peter Wilhelmsson, ${ }^{1}$ Linda Fryland, ${ }^{2}$ Mats Haglund, ${ }^{4}$ Andreas Matussek, ${ }^{6}$ Jan Ernerudh, ${ }^{2}$ Sirkka Vene, ${ }^{7}$ Dag Nyman, ${ }^{8}$ Åshild Andreassen, ${ }^{9}$ Pia Forsberg, ${ }^{3,5}$ and Per-Eric Lindgren ${ }^{1,6}$

${ }^{I}$ Division of Medical Microbiology, Department of Clinical and Experimental Medicine, Linköping University, Linköping, Sweden

${ }^{2}$ Division of Clinical Immunology, Department of Clinical and Experimental Medicine, Linköping University, Linköping, Sweden

${ }^{3}$ Division of Infectious Diseases, Department of Clinical and Experimental Medicine, Linköping University, Linköping, Sweden

${ }^{4}$ Clinic of Infectious Diseases, County Hospital, Kalmar, Sweden

${ }^{5}$ Clinic of Infectious Diseases, County Council of Östergötland, Linköping, Sweden

${ }^{6}$ Medical Services, Department of Microbiology, County Hospital Ryhov, Jönköping, Sweden

${ }^{7}$ Swedish institute for Communicable Disease Control, Stockholm, Sweden

${ }^{8}$ Åland central hospital, Mariehamn, Åland, Finland

${ }^{9}$ Norwegian Institute of Public Health, Oslo, Norway

*Corresponding author

Pontus Lindblom

Linköping University

Department of Clinical and Experimental Medicine

Division of Medical Microbiology

58185 Linköping

Sweden

Phone: (+46) 101032056

Fax: (+46) 101034789

Email: Pontus.lindblom@liu.se 


\begin{abstract}
The risk of tick-borne encephalitis virus (TBEV) infection after a tick-bite remains largely unknown. To address this, we investigated the presence of TBEV in ticks detached from humans in an attempt to relate viral copy number, TBEV subtype, and tick feeding-time with the serological and clinical response of the tick-bitten participants. Ticks, blood samples and questionnaires were collected from tick-bitten humans at 34 primary health care centers in Sweden and the Åland Islands (Finland). 2,167 ticks were received from 1,886 tick-bitten persons in 2008 - 2009. Using a multiplex quantitative real-time PCR, five TBEV infected ticks (overall prevalence $0.23 \%$, copy range $<4 \times 10^{2}-7.7 \times 10^{6}$ per tick) were found. One unvaccinated person bitten by a tick containing $7.7 \times 10^{6}$ TBEV copies experienced symptoms. Another unvaccinated person bitten by a tick containing $1.8 \times 10^{3}$ TBEV copies developed neither symptoms nor TBEV antibodies. The remaining three persons were protected by vaccination. In contrast, despite lack of TBEV in the detached ticks, two persons developed antibodies against TBEV, one of whom reported symptoms. Overall, a low risk of TBEV infection was observed, and too few persons got bitten by TBEV infected ticks to draw certain conclusions regarding the clinical outcome in relation to duration of blood meal and virus copy number. However, this study indicates that an antibody response may develop without clinical symptoms, that a bite by an infected tick not always leads to antibody response or clinical symptoms, and a possible correlation between virus load and the tick feeding time.
\end{abstract}

\title{
Keywords
}

TBEV; Ixodes ricinus; Clinical symptoms; Serology; real-time PCR 


\section{Introduction}

Tick-borne encephalitis (TBE) is a serious acute central nervous system (CNS) disease in Europe and Asia, which can lead to death or long term morbidity. It has been found that as much as $46 \%$ of patients may suffer from postencephalitic symptoms (Haglund et al., 1996; Günther et al., 1997; Kaiser, 1999; Mickiene et al., 2002; Haglund and Günther, 2003; Kaiser, 2011). The TBE virus (TBEV) belonging to the genus Flavivirus is a positive-sense single stranded RNA virus transmitted by ticks of the species Ixodes. TBEV caused at least 7,200 human cases of TBE in Europe during 2010, of which half occurred in Russia (Süss, 2011). The mean number of human TBE cases per year in Sweden has increased from 63 in 19851999 to 184 in 2007-2010. In 2011, 284 TBE cases (3 per 100,000 inhabitants) was registered (Jaenson et al., 2012), and a few more (287 cases) 2012 . In contrast, on the Åland Islands (Finland), human TBE has decreased from over 20 annual cases in 2000-2002 (77 per $100,000)$ to $3-13$ annual cases in 2006-2010 (11 - 50 per 100,000). A vaccination program initiated in 2006 covering the whole population from seven years and older on the Åland Islands is the likely explanation for this decrease (Jääskeläinen et al., 2011).

In Sweden and on the Åland Islands, only the European (Eu) subtype of TBEV, spread by the tick species Ixodes ricinus, has been found so far. Across the Baltic sea, in Estonia, Latvia and Lithuania, also the Far-Eastern (FE) and Siberian (Sib) TBEV subtypes, spread by the tick species Ixodes persulcatus, are present (Golovljova et al., 2004). Notably, a hotspot of TBEVSib was discovered 2004 in the Kokkola Archipelago (Finland), $100 \mathrm{~km}$ east of the Swedish coast (Jääskeläinen et al., 2006).

Several studies from different European countries have investigated the prevalence of TBEV in field-collected, unfed ticks, while only a few have investigated the TBEV prevalence in 
ticks that have bitten humans (Supplementary Table S1). In three studies from Germany, a significantly higher prevalence of TBEV was found in ticks detached from humans than in field-collected ticks from the same area (Klaus et al., 2010; Süss et al., 2006, 2004). There is also a report from Karelia in Russia showing a similar pattern (Kotovich, 2012). Unlike the Borrelia spirochete, which requires 24-48 h for transmission to mice (Crippa et al., 2002), TBEV has been shown to be transmitted to mice directly after tick attachment due to the presence of virus in the tick salivary glands (Alekseev et al., 1996). However, regarding the risk of TBEV transmission to humans and the clinical outcome, the importance of factors such as the tick life stage, tick feeding-time, virus copy number and virus subtype remains to be clarified.

To investigate the overall risk of contracting TBEV infection through a tick-bite, a comprehensive study, denoted the tick-borne diseases (TBD) STING study, was set up. The aim was to assess the prevalence of TBEV in ticks detached from humans and to evaluate if TBEV copy number, subtype, the life stage of the tick, and the tick feeding-time influence the risk of virus transmission and the development of symptomatic or asymptomatic infections. In order to optimally determine the presence, amount and subtype of TBEV in ticks, a multiplex quantitative real-time PCR assay based on two existing assays (Schwaiger and Cassinotti, 2003; Gäumann et al., 2010) was established.

\section{Materials \& Methods}

\section{Study Design}


Collection of ticks, blood samples and questionnaires from tick-bitten humans were done in collaboration with 34 primary health care centers (PHCs) during 2008 and 2009. The PHCs were located in the Åland Islands (Finland) and in three regions of Sweden (Fig. 1). All the included regions are TBE endemic except Northern Sweden, but with a patchy distribution. At each PHC, local advertisements through different media were used to publicize the study in order to recruit tick-bitten volunteers, 18 years and older. At inclusion, after the participant had provided informed consent, the tick/ticks that had bitten the participant were collected, blood samples were taken, and a questionnaire was completed. Follow-up blood samples and questionnaires were collected from each participant three months after the tick-bite. During the three-month study period, the participants were also asked to collect any additional ticks that had bitten them. Ticks and blood samples were sent within three days to Linköping University, where they were frozen at $-70{ }^{\circ} \mathrm{C}$ until analysis. If a participant developed clinical symptoms, they were asked to visit the PHC where additional samples were taken. Ethical approval for the TBD STING study was granted by the Regional Ethics Committee in Linköping (M132-06), and by the local Ethics Committee of the Åland Health Care, 2008-0523.

\section{Tick photo and measurement}

Each tick was photographed and measured dorsally and ventrally, using a USB-microscope (Dino-Lite Long AM4013TL, AnMo Electronics Corp., Taiwan) to determine species, lifestage, and estimate feeding duration using coxal and scutal indices (Gray et al., 2005).

\section{RNA-extraction and cDNA synthesis}


Ticks were homogenized individually by bead-beating in $2 \mathrm{ml}$ safe-lock microcentrifuge tubes (Eppendorf AG, Hamburg, Germany) with a $5 \mathrm{~mm}$ stainless steel bead (Qiagen, Hilden, Germany) in $450 \mu 1$ RLT-buffer (Qiagen), supplemented with 1\% $\beta$-mercaptoethanol (Qiagen), using a TissueLyser (Qiagen), 2 minutes at $25 \mathrm{~Hz}$. After centrifugation at 20,000 g for 3 minutes, $400 \mu$ l supernatant was transferred to new microcentrifuge tubes for RNA extraction, using MagAttract ${ }^{\circledR}$ RNA Tissue Mini M48 kit (Qiagen) in a Biorobot M48 workstation (Qiagen), using a $50 \mu$ l elution volume. Each batch of 48 samples consisted of 46 ticks, one positive control ( $5 \mu$ l inactivated TBEV strain K23, Encepur®, Chiron Vaccines, Marburg, Germany and $5 \mu 1$ of Borrelia burgdorferi sensu stricto B31 ATCC 35210 (108 cells/ml) ) and one negative control $\left(\mathrm{H}_{2} \mathrm{O}\right)$ extracted simultaneously. The RNA was reversetranscribed to cDNA using illustra Ready-To-Go RT-PCR Beads (GE-healthcare, Amersham Place, UK). Twenty $\mu 1$ RNA and $10 \mu \mathrm{l} \mathrm{pd}(\mathrm{N}) 6$ random hexamer primers $(0.25 \mu \mathrm{g} / \mu \mathrm{l})$ were incubated for 5 minutes at $97^{\circ} \mathrm{C}$, then mixed with one RT-PCR bead dissolved in $20 \mu \mathrm{l}$ RNAse free water. The mixture was incubated for 30 minutes at $42^{\circ} \mathrm{C}$, followed by 5 minutes at $97^{\circ} \mathrm{C}$, producing $50 \mu \mathrm{l} \mathrm{cDNA}$. Pipetting was done using a CAS-1200 pipetting robot (Corbett robotics Pty Ltd., Queensland, Australia) and incubation using a PTC-100 thermal cycler (MJ Research, MA, USA).

\section{Detection and quantification of TBEV using real-time PCR}

To evaluate detection of a wide range of TBEV strains, dilution series were made for 12 TBEV strains including all three TBEV subtypes. The detection limit for the individual strains using two published TaqMan ${ }^{\text {TM }}$ real-time PCR assays (Schwaiger and Cassinotti, 2003; Gäumann et al., 2010) was compared. The two assays were also evaluated in combination as a multiplex TaqMan ${ }^{\mathrm{TM}}$ assay. The signals from the two assays could be distinguished as the 
probes were labeled with different fluorescent reporters (FAM and HEX). The TBEV strains used for the evaluation were K23(Eu) (Ecker et al., 1999); 93-3511(Eu), Lat 1-96(Sib), Sofjin (FE) (Haglund et al., 2003); Lat 12849(Eu) (Mickiené et al., 2001); Est 3051(Eu), Est 3509(Eu), Est 3053(Eu), Est 3476(Eu), Est 54(Sib), Est 3535(Sib) (Golovljova et al., 2004) and the strain $9 * 000801(\mathrm{Eu})$, isolated from human blood and passaged once through suckling mouse brain in Sweden year 2000 (unpublished results). The real-time PCR reactions were set up using a CAS-1200 pipetting robot (Corbett robotics). Each $20 \mu 1$ reaction consisted of 10 $\mu 1$ Maxima ${ }^{\circledR}$ Probe qPCR Master Mix (Fermentas, St. Leon-Rot, Germany), $200 \mathrm{nM}$ each of primers and probes: F-TBE 1, R-TBE 1, TBE-Probe-WT, TBEE-F6, TBEE-R2, TBEE-P4 (Table 1), $2 \mu 1$ template and RNAse free water up to $20 \mu 1$. For quantification of TBEV, a serial dilution of a plasmid standard was used. This was a synthetic plasmid containing the target sequences of the multiplex TaqMan assay, spanning nucleotides 1,319-1,426 and $11,044-11,134$, synthesized and cloned in a pUC57 vector (Genscript USA Inc, NJ). The source sequence was strain Neudoerfl U27495. The PCR reactions were performed on a Rotor-Gene 6000 (Qiagen) using an activation step at $95^{\circ} \mathrm{C}$ for $5 \mathrm{~min}$, and 45 cycles of $95^{\circ} \mathrm{C}$, $10 \mathrm{~s}$ and $60^{\circ} \mathrm{C}, 60 \mathrm{~s}$.

\section{Sequencing of the E-gene and phylogenetic analysis}

For all ticks positive for TBEV according to the real-time PCR assay, sequencing of the Egene was attempted. The E-gene was amplified using a nested PCR with primers E OF, E OR, E IF, E IR (Table 1), as previously described (Melik et al., 2007). Nucleotide sequencing of PCR products obtained was performed by Macrogen Inc. (Seol, South Korea), using BigDye chemistry. Sequences were analyzed using BioEdit software (v.7.0.5.3, Tom Hall, Ibis 
Table 1. Primers and probes used for analysis of TBEV.

\begin{tabular}{|c|c|c|c|}
\hline Name (ref) & Sequence $\left(5^{\prime} \rightarrow 3^{\prime}\right)$ & Genome region $^{\mathrm{e}}$ & Amplicon length (bp) \\
\hline F-TBE $1^{\mathrm{a}}$ & GGG CGG TTC TTG TTC TCC & $11,054-11,071$ & \\
\hline $\mathrm{R}-\mathrm{TBE} 1^{\mathrm{a}}$ & ACA CAT CAC CTC CTT GTC AGA CT & $11,099-11,121$ & 68 \\
\hline TBE-Probe-WT ${ }^{\mathrm{a}}$ & (FAM) TGA GCC ACC ATC ACC CAG ACA CA (BHQ1) & $11,073-11,095$ & \\
\hline TBEE-F6 ${ }^{\mathrm{b}}$ & GGC TTG TGA GGC AAA AAA GAA & $1,329-1,349$ & \\
\hline TBEE-R2 $2^{\mathrm{b}}$ & TCC CGT GTG TGG TTC GAC TT & $1,397-1,416$ & 88 \\
\hline TBEE-P4 ${ }^{b}$ & $(H E X)$ AAG CCA CAG GAC ATG TGT ACG ACG CC (BHQ1) & $1,349-1,374$ & \\
\hline $\mathrm{E} \mathrm{OF}$ & CGG GGA GGG GAC ACA AAT GG & $785-804$ & \\
\hline $\mathrm{E} \mathrm{OR}^{\mathrm{c}}$ & AGG CAT AGT TGT CAT ACC & $2,560-2,543$ & 1,759 \\
\hline $\mathrm{E} \mathrm{IF}^{\mathrm{c}}$ & GTT GTG CTC CTG TGT TTG GC & 940-959 & \\
\hline $\mathrm{E} \mathrm{IR}^{\mathrm{c}}$ & CTC CAT TCG TTC CGT GTC & $2,496-2,479$ & 1,540 \\
\hline TBE $320 F^{d}$ & GGG AGC GCA AAA CTG GAA & $1,662-1,679$ & \\
\hline TBE $373 R^{d}$ & (Biotin) TGA GGA GCC CCA AAT TCA AC & $1,696-1,715$ & 54 \\
\hline TBE 339 probe $^{\mathrm{d}}$ & $(F A M)$ AAC GCA GAA AGA C (BHQ1) & $1,681-1,693$ & \\
\hline F-16sIxodes ${ }^{a}$ & AAA AAA ATA CTC TAG GGA TAA CAG CGT AA & $254-282^{f}$ & 98 \\
\hline R-16sIxodes ${ }^{\mathrm{a}}$ & ACC AAA AAA GAA TCC TAA TCC AAC A & $327-351^{\mathrm{f}}$ & \\
\hline
\end{tabular}

FAM: 6-carboxy-fluorescine, HEX: 6-carboxy-hexachlorofluorescein, BHQ: Black Hole Quencher.

${ }^{\mathrm{a}}$ Schwaiger and Cassinotti, 2003, ${ }^{\mathrm{b}} \mathrm{Gäumann}$ et al., 2010, ${ }^{\mathrm{c}}$ Melik et al., 2007, ${ }^{\mathrm{d}}$ Andreassen et al., 2012.

In reference to: ${ }^{\mathrm{e}} \mathrm{TBEV}$ strain Neudoerfl (U27495), ${ }^{\mathrm{f}} 16 \mathrm{~S}$ ribosomal RNA of I. ricinus (Z97882) 
Therapeutics, Carlsbad, CA) after multiple sequence alignment using ClustalW2 (Larkin et al., 2007). Phylogenetic analysis was done with the MEGA5 software (Tamura et al., 2011).

\section{Pyrosequencing to confirm TBEV prevalence in ticks}

To confirm TBEV presence in ticks detected by real-time PCR, but with too few copies to sequence the E-gene as described above, we also performed pyrosequencing using the primers and probe TBE 320F, TBE 373R, TBE 339 (Table 1), targeting the E-gene (Andreassen et al., 2012).

\section{Internal control}

In order to assure the reproducibility of the RNA extraction and cDNA synthesis, and to prevent false-negative results, at least 2 randomly selected tick-samples in each 46-tick extraction batch were analyzed for the 16s rRNA gene of the Ixodes ricinus tick, with a realtime PCR assay (Wilhelmsson et al., 2010), using the forward primer F-16sIxodes and the reverse primer R-16sIxodes (Table 1).

\section{Clinical data collection}

The first questionnaire, answered by the study participants at enrollment, included geographical location of the tick-bite, previous tick-borne infections, general health status, and vaccination status for TBE, Yellow fever and Japanese encephalitis. The second questionnaire covered information about new tick bites during the three-month study period, general health during the period, symptoms possibly associated with TBEV (headache, 
fatigue, fever, neck pain, loss of appetite, nausea, weight loss, vertigo, concentration difficulties, radiating pain, myalgia/arthralgia, and numbness), and whether or not the participant had attended their PHC because of these symptoms. Medical records from participants, all of whom attended health care for symptoms possibly associated with TBE or other TBDs during the study period, were examined in detail by physicians with long-time clinical experience of TBDs.

\section{TBEV Serology}

All three-month serum samples from the tick-bitten persons were screened for TBEV IgG antibodies. Positive samples, together with the corresponding initial samples, were selected for further analysis for both $\operatorname{IgG}$ and $\operatorname{IgM}$ to assess whether seroconversion had occurred during the study period. Samples from vaccinated participants and participants with a history of TBE infection were excluded. Tests were performed using Enzygnost anti-TBE IgG and IgM (Siemens, Erlangen, Germany) on a BEP 2000 Advance® System (Siemens). Seroconversion, indicating a current infection, was defined as a change from seronegative to seropositive between the initial and the three-month blood sample. Sera from persons with seroconversion were further confirmed using Immunozym ${ }^{\circledR}$ FSME (TBE) IgG and IgM (PROGEN Biotechnik GmbH, Heidelberg, Germany) and a rapid fluorescent focus inhibition test (RFFIT), for detection of TBEV neutralizing antibodies (Vene et al., 1998). In addition, persons with seroconversion were interviewed to find out whether they had been vaccinated during the study period.

\section{Results}




\section{Evaluation of TBEV real-time PCR}

The primers and probes of two previously published real-time PCR assays, Schwaiger and Cassinotti, 2003 and Gäumann et al., 2010, were evaluated both separately and combined into a multiplex TaqMan ${ }^{\mathrm{TM}}$ assay on $12 \mathrm{TBEV}$ strains. The two assays differed in detection limits for the tested strains. For the TBEV-Eu strains 93-3511, Est 3051, Est 3476, Est 3509 and Est 3053, the Gäumann et al. assay was found to be more sensitive, as it was able to detect $8,8,8$, 16 and 64 times more diluted samples respectively than the Schwaiger \& Cassinotti assay. In contrast, the latter assay was more sensitive in the detection of the TBEV-Sib and TBEV-FE strains, which the Gäumann et al. assay is not designed to detect, but also more sensitive by a dilution factor of 8 for the TBEV-Eu strain $9 * 000801$. For the two TBEV-Eu strains Lat 12849 and K23, the detection limit was equivalent. Combining the two assays into a multiplex assay did not reduce the performance in detection and quantification compared to running the two assays separately. Regarding the detection limit, 10 copies per reaction of the plasmid standard used were readily detectable in both the singleplex and multiplex assay setup. Thus, the multiplex setup was used for analysis of all tick-samples.

\section{Ticks from humans}

From May 2008 to December 2009 a total of 2,167 ticks that had bitten 1,886 humans, were received from the four included regions. A number of participants $(n=216)$ were bitten by more than one tick. The ticks, 91 larvae, 1,517 nymphs, 498 adult females and 15 adult males, were morphologically determined to belong to the species Ixodes ricinus (Table 2). Forty-six of the 2,167 ticks $(2.1 \%)$ were so fragmented that neither life-stage nor species could be determined morphologically, but they were included since Ixodes 16S rRNA genes were detected in all of them. The internal control, Ixodes 16S rRNA gene was detected in all 
randomly selected samples tested $(n=333)$. The threshold cycle $\left(\mathrm{C}_{T}\right)$ range differed depending on life-stage of the ticks (adults 9-21, median 13; nymphs 9-21, median 13; larvae 12-23, median 16). For the 46 fragmented ticks, where life-stage could not be determined morphologically, the $\mathrm{C}_{\mathrm{T}}$ range was $10-37$, median 21 . The median feeding duration inferred from tick dimensions (Gray et al., 2005) for all the nymphs was 33 hours, and for the adult females 37 hours.

Table 2. Ticks detached from humans in Sweden and the Åland Islands (Finland).

\begin{tabular}{llllllll}
\hline Region & $\begin{array}{l}\text { Total } \\
\text { ticks, } \mathrm{n}\end{array}$ & $\begin{array}{l}\text { Adult } \\
\text { female } \%\end{array}$ & $\begin{array}{l}\text { Adult } \\
\text { male } \%\end{array}$ & $\begin{array}{l}\text { Nymph } \\
\%\end{array}$ & $\begin{array}{l}\text { Larvae } \\
\%\end{array}$ & $\begin{array}{l}\text { ND } \\
\%\end{array}$ & $\begin{array}{l}\text { TBEV }+ \\
\text { ticks, n }(\%)\end{array}$ \\
\hline $\mathrm{A}$ & 554 & 20.8 & 0.2 & 74.0 & 2.7 & 2.3 & $1(0.2)$ \\
$\mathrm{B}$ & 794 & 32.3 & 1.1 & 63.6 & 0.6 & 2.4 & 0 \\
$\mathrm{C}$ & 19 & 47.4 & 0 & 47.4 & 5.2 & 0 & $1(0.1)^{\mathrm{a}}$ \\
$\mathrm{D}$ & 800 & 14.7 & 0.6 & 74.2 & 8.7 & 1.8 & $3(0.4)$ \\
\hline Total & 2,167 & 23.0 & 0.7 & 70.0 & 4.2 & 2.1 & $5(0.2)$ \\
\hline
\end{tabular}

A: South Sweden, B: Middle Sweden, C: North Sweden, D: the Åland Islands, n: number. ND: developmental stage could not be determined due to the condition of the ticks.

${ }^{\text {a }}$ The person from northern Sweden got the tick-bite in Saringe in middle Sweden.

\section{Tick-bitten persons}

1,886 tick-bitten persons were included, of whom 1,670 (89\%) attended the three-month follow-up. 578 of the 1,670 (35\%) persons reported additional tick-bites during the study period. There were 1,202 (64\%) female participants (age 19 - 92, median 61), and 684 (36 \%) male participants (age 19 - 89, median 65). The TBEV vaccination coverage varied (Southernmost Sweden $17 \%$, South Central Sweden $31 \%$, Northern Sweden $22 \%$, and Åland Islands $88 \%$ ), as reported by the participants in the questionnaires. Furthermore, 18 participants reported to have had TBE previously, of which 12 were from the Åland Islands (Table 3). 
Table 3. Number of participants from different regions, received three-month samples, history of previous TBE, TBEV vaccination status, and TBEV seroconversion.

\begin{tabular}{lllllll}
\hline Region & $\begin{array}{l}\text { Persons } \\
\mathrm{n}\end{array}$ & \multicolumn{2}{l}{$\begin{array}{l}\text { 3-month samples } \\
\mathrm{n},(\% \text { loss })\end{array}$} & $\begin{array}{l}\text { Previous TBE }^{\mathrm{a}} \mathrm{n},(\%) \\
\mathrm{TBEV} \text { vacc. }^{\mathrm{a}}\end{array}$ & $\begin{array}{l}\text { Seroconversion } \\
\mathrm{n},(\%)\end{array}$ \\
\hline $\mathrm{A}$ & 507 & 409 & $(19)$ & $2(0.4)$ & $85(17)$ & 2 \\
$\mathrm{~B}$ & 746 & 642 & $(14)$ & $4(0.5)$ & $229(31)$ & 0 \\
$\mathrm{C}$ & 18 & 15 & $(17)$ & $0(0.0)$ & $4(22)$ & 0 \\
$\mathrm{D}$ & 615 & 604 & $(1.8)$ & $12(2.0)$ & $542(88)$ & 0 \\
\hline Total & 1,886 & $1,670(12)$ & $18(1.0)$ & $860(46)$ & 2 \\
\hline
\end{tabular}

A: South Sweden, B: Middle Sweden, C: North Sweden, D: the Aland Islands, n: number. ${ }^{a}$ According to self-reported health history through the questionnaires.

${ }^{\mathrm{b}}$ Anti-TBEV IgG change from seronegative to seropositive during the study period without vaccination.

\section{TBEV in ticks from humans}

Five out of 2,167 investigated ticks (0.23\%), all nymphs, contained TBEV (Table 4). One tick had a virus load of 7.7 million copies (feeding-time $>60 \mathrm{~h}$ ), the other four varied from less than 400 to 4,200 virus copies (feeding-time $0-36$ h). The tick with the highest viral load (Table 4, No. 1) came from Saringe $\left(60^{\circ} 0.38^{\prime} \mathrm{N}, 18^{\circ} 3.3^{\prime} \mathrm{E}\right), 30 \mathrm{~km}$ north-east of Uppsala in Sweden. Another tick with TBEV (Table 4, No. 3) came from either Värnanäs $\left(56^{\circ} 30^{\prime} \mathrm{N}, 16^{\circ} 9.3^{\prime} \mathrm{E}\right)$ or Harbymo $\left(56^{\circ} 41^{\prime} \mathrm{N}, 16^{\circ} 9.6^{\prime} \mathrm{E}\right)$ near Kalmar, in Sweden. The remaining three ticks with TBEV came from the Åland Islands (Finland), and the persons reported to have acquired the ticks in Torp, Jomala $\left(60^{\circ} 8.0^{\prime} \mathrm{N}, 19^{\circ} 54^{\prime} \mathrm{E}\right)$, Kökar $\left(59^{\circ} 55^{\prime} \mathrm{N}\right.$, $\left.20^{\circ} 55^{\prime} \mathrm{E}\right)$, and Geta, Gröndal $\left(60^{\circ} 25^{\prime} \mathrm{N}, 19^{\circ} 54^{\prime} \mathrm{E}\right)$, respectively (Table 4, Nos. 2, 4 and 5). All five ticks were confirmed to be infected by TBEV using pyrosequencing, but nested PCR for amplification of the E-gene was only successful for the TBEV strain with the highest copy number (Saringe-2009, deposited in GenBank, accession number KC469073). The E-gene sequence of Saringe-2009 was closely related (Figure 2) to the TBEV strain Toro-2003 (DQ401140), which was found in ticks collected at the island Torö $\left(58^{\circ} 49^{\prime} \mathrm{N}, 17^{\circ} 50^{\prime} \mathrm{E}\right)$ in the Stockholm archipelago in 2003 (25), differing in ten base pairs and only one amino acid in 
Table 4. Five ticks (all nymphs) positive for TBEV detached from humans.

\begin{tabular}{lllll|llll}
\hline \multicolumn{4}{c|}{ Tick containing TBEV } & \multicolumn{3}{c}{ Person bitten by tick containing TBEV } \\
\hline No. & Region & $\begin{array}{l}\text { Feeding- }^{\text {Feedine }} \\
\text { time }^{\mathrm{a}}(\mathrm{h})\end{array}$ & $\begin{array}{l}\text { TBEV } \\
\text { copies }^{\mathrm{b}}\end{array}$ & $\begin{array}{l}\text { Tick co- } \\
\text { infection }^{\mathrm{c}}\end{array}$ & $\begin{array}{l}\text { Month of } \\
\text { tick-bite }\end{array}$ & $\begin{array}{l}\text { IgG sero- } \\
\text { conversion }\end{array}$ & $\begin{array}{l}\text { TBEV vacc. } \\
\text { before study }\end{array}$ & Symptoms \\
\hline 1 & $\mathrm{C}$ & $>60$ & $7.7 \times 10^{6}$ & Borrelia & July & $\mathrm{ND}^{\mathrm{d}}$ & No & Yes \\
2 & $\mathrm{D}$ & $24-36$ & $4.2 \times 10^{3}$ & No & Aug. & No & Yes & Yes \\
3 & $\mathrm{~A}$ & $12-24$ & $1.8 \times 10^{3}$ & No & June & No & No & No \\
4 & $\mathrm{D}$ & $<12$ & $<4 \times 10^{2}$ & Borrelia & Aug. & No & Yes & No \\
5 & $\mathrm{D}$ & $<12$ & $<4 \times 10^{2}$ & Anaplasma & Sept. & No & Yes & No \\
\hline
\end{tabular}

A: South Sweden, B: Middle Sweden, C: North Sweden, D: the Aland Islands, ND: not determined.

${ }^{a}$ Based on measurements of tick dimensions (Gray et al., 2005).

${ }^{\mathrm{b}} \mathrm{TBEV}$ copies in the tick calculated from a detection limit of five gene copies per PCR reaction.

${ }^{\mathrm{c}}$ (Wilhelmsson et al. unpublished; Kozak et al. unpublished)

${ }^{\mathrm{d}}$ The person did not attend follow-up. 
the E-gene. The material collected in the TBD STING study was not only investigated for TBEV, but also for the tick-borne pathogens Borrelia spp., Anaplasma phagocytophilum, and Rickettsia spp. reported elsewhere (Wilhelmsson et al. unpublished, Kozak et al.

unpublished). When screening for other tick-borne pathogens in the five TBEV infected ticks, Borrelia was found in two of the ticks, and Anaplasma in one (Table 4).

\section{Clinical outcome for persons bitten by TBEV infected ticks}

The three persons bitten by TBEV infected ticks on the Åland Islands had been vaccinated against TBEV, but not the two persons from Sweden. One of the three TBEV vaccinated persons (Table 4, No 2) bitten by a TBEV infected tick reported fatigue and vertigo during the study period. However, this 85 -year-old woman had visited the PHC one month before inclusion with an erythema around a tick-bite, and reported swollen lymph nodes at inclusion. She also got bitten by three more ticks during the study and was seropositive for Borrelia. A definitive clinical diagnosis was not established but ongoing Lyme borreliosis was suspected in the routine clinical diagnosis on Åland Islands.

The unvaccinated person from Kalmar, Sweden, was bitten by a tick containing 1,800 virus copies but did not develop TBEV antibodies or clinical signs of infection (Table 4, No 3). The other person, a healthy 72-year-old man, who was bitten in Saringe by a tick containing 7.7 million TBEV copies and co-infected with Borrelia, did not attend the three-month follow-up (Table 4, No 1). He was contacted two years later and we were informed that he got a few days of fever and headache one week after the tick-bite and about one month after the tickbite he visited the PHC because of blurred vision and trouble with eye sight focusing. This could be mild symptoms of CNS involvement from a TBEV infection. After that he had been feeling well and had received vaccination for TBEV with three doses, starting one year after 
the tick-bite. The first serum sample taken after the tick-bite was negative in both TBEV IgM and IgG antibodies. A two-year sample, taken after the contact tested negative in IgM but strongly positive in $\mathrm{IgG}$ antibodies (Immunozym ${ }^{\circledR}$ FSME). Whether the seroconversion was caused by a natural infection or the vaccination could not be established.

\section{Persons with TBEV antibody seroconversion}

13 of the 1,670 persons who delivered a three-month follow-up sample had anti-TBEV IgG seroconversion during the study period, as confirmed by Enzygnost anti-TBE IgG, Immunozym FSME IgG and RFFIT for detection of TBEV neutralizing antibodies (data not shown). However, when interviewed, 11 of the 13 individuals reported receiving a TBEV vaccination during the study period. Thus, only two of $1,670(0.1 \%)$ participants had a seroconversion that could not be attributed to vaccination (Table 3). No TBEV was detected in the ticks from these two persons. One had no symptoms at all and did not report any additional tick-bites. However, the other person reported headache, fatigue, neck pain, vertigo, and concentration difficulties starting after several additional tick-bites. Six additional blood-fed nymphs were received from this patient, all TBEV negative. Interestingly, the first tick contained Rickettsia sp. Both of the persons who seroconverted reported being tick-bitten in the same geographical region, near Vinslöv $\left(56^{\circ} 6^{\prime} \mathrm{N}, 13^{\circ} 55^{\prime} \mathrm{E}\right)$ in Southern Sweden.

\section{Discussion}

Only five of 1,886 participants were bitten by a TBEV infected tick. Two of these persons were not vaccinated against TBEV at the time of the tick-bite. The first person, bitten by a TBEV infected tick containing 7.7 million copies (feeding-time $>60 \mathrm{~h}$ ), experienced 
symptoms suggesting a possible first viremic phase of a TBEV infection and perhaps mild CNS involvement in the form of distorted eye sight. Unfortunately, no seroconversion could be determined due to no three-month follow-up sample given. It could be expected that a bite from a tick with a high viral load, as in the present case, leads to a higher risk of infection and clinical symptoms and in Russia, for Ixodes persulcatus ticks, the frequency of human contact with highly TBEV infected ticks as compared to ticks with low virus levels, have been shown to be the main factor determining TBE incidence (Korenberg and Kovalevskii YuV, 1995). Notably, the other unvaccinated person that was bitten by a tick containing relatively few $(1,800)$ copies of TBEV (feeding-time 12-24 h), neither developed any symptoms nor antibodies. Either the virus did not get transmitted to the tick bitten person or there were too few virus copies to elicit an immune response involving antibody production or establish an infection. By way of comparison, studies in mice bitten by ticks inoculated with TBEV, shows that virus transmission occurs soon after tick attachment and with increasing virus titers in the tick saliva during the first three days of feeding (Alekseev et al., 1996). A recent study also reports that TBEV replication takes place during tick feeding (Belova et al., 2012). However, it is possible that transmission dynamics for mice and humans may differ and also in that of naturally infected ticks as opposed to in vitro inoculated ticks.

The three other persons bitten by ticks containing TBEV were all vaccinated. Two of those ticks contained $<400$ virus copies (feeding-time $<12$ h), and one tick contained 4,200 virus copies (feeding-time 24-36 h).

Interestingly, we did not observe CNS-involvement in any of the participants bitten by the TBEV infected ticks. Two other persons had an anti-TBEV IgG seroconversion during the three-month follow-up, despite that no TBEV was detected in the ticks that had bitten them. Either the virus copy number was below the detection limit, contradicting the speculations above, or they been bitten by additional ticks that they did not notice and thus didn't send in 
to us. One of the seroconverting persons developed symptoms, possibly caused by TBEV infection, but got well and did not contact a PHC.

Considering the high TBEV prevalence observed in ticks detached from humans in previous studies (Klaus et al., 2010; Süss et al., 2006, 2004), we expected to find a higher overall prevalence than the observed $0.23 \%$ (5 of 2167 ticks). Our prevalence in ticks detached from humans was in the same range as in field-collected ticks from Sweden $(0.1-0.42 \%)$ (Brinkley et al., 2008). However, in that study the field-collection of ticks was done at local hotspots for TBEV, where several patients had contracted TBEV. In our study, we could not influence where our participants got bitten, and since we only examined ticks detached from humans (with the further purpose to evaluate transmission frequency and clinical outcome), we cannot assess whether the TBEV prevalence found is higher than in questing ticks from the corresponding areas.

There are two suggested hypotheses why ticks detached from humans may have a higher prevalence of TBEV than questing ticks. The first is that TBEV is equally prevalent in questing ticks but the virus copy number is below the detection limit (Süss et al., 2006). During the blood meal the virus starts to replicate, either because of the introduction of the mammalian blood cells that the virus can replicate in (Senigl et al., 2006), or because TBEV replication gets triggered by the increased temperature (Elväng et al., 2011). The second hypothesis is that TBEV infected ticks are more aggressive than uninfected ticks. Both of these hypotheses are supported by a recent study reporting that the titer of TBEV-Eu strain Absettarov increased by a factor of 500 in in vitro inoculated I. ricinus ticks after 15 hours of feeding (Belova et al., 2012). They also reported that TBEV infected ticks became more aggressive, active and had a higher tolerance for the commonly used insect repellent DEET (N,N-Diethyl-meta-toluamide). In contrast, a previous study on the tick species Ixodes persulcatus reported no overrepresentation of bites by TBEV infected ticks (Korenberg et al., 
2001), so there might be a difference between the two tick species or the observed difference may stem from the methods used.

Our results suggest that there may be a connection between the tick feeding-time and the amount of TBEV found in the ticks detached from humans. This suggests that the time of blood feeding may be an important factor determining the clinical outcome if bitten by a TBEV infected tick, and perhaps even the risk of transmission as a whole, as suggested by the person bitten by a TBEV infected tick that got neither symptoms nor antibody response. That would make the risk of TBEV transmission and clinical outcome more in line with the dynamics of Borrelia transmission where the tick feeding-time is an important factor (Kahl et al., 1998).

However, since we only had five TBEV infected ticks in this study, no certain conclusions can be made regarding the relationship between virus copies in the tick and duration of the blood meal, and for the same reason, regarding the risk of clinical outcome in relation to duration of blood meal and the virus copy number in the tick.

It is known from previous studies that the overall prevalence of TBEV in ticks usually is low (Table S1), and also that from Sweden and Norway the majority of TBEV infected ticks have a virus amount around the detection limit (Andreassen et al., 2012; Brinkley et al., 2008). Also considering that several new foci of TBEV-Sib have been found in Finland close to the Swedish coast (Jääskeläinen et al., 2006, 2010; Alekseev et al., 2007; Tonteri et al., 2011), and that all three subtypes of TBEV are present in the Baltic countries (Bormane et al., 2004; Golovljova et al., 2004; Han et al., 2005), we wanted to make sure that we had an assay with both high specificity and as high sensitivity as possible for all three TBEV subtypes. To accomplish this, we evaluated two previously reported TaqMan assays (Schwaiger and Cassinotti, 2003; Gäumann et al., 2010). These assays were found to differ in sensitivity for 
the 12 TBEV strains that we tested, representing all three TBEV subtypes. The optimal solution was attained by combining the primers and probes from the two assays into a multiplex assay. In this way a cost- and time efficient method was established, without loss in sensitivity for detecting the various TBEV strains. As there are strain-specific sensitivity differences in the two assays and the signals can be distinguished by the color of the labeled probe, it may be possible to use this assay as a preliminary genotyping method based on the relative $\mathrm{Ct}$ values for each assay.

The high median age of the tick-bitten persons participating in our study (65 years for males and 61 years for females), clearly exceeded the mean age in Sweden for persons over 18 years of age (48 years for males and 50 years for females according to www.scb.se). A reason may be that older people are more willing to spare the time and effort needed to take part in studies, or that the communication channels used to inform about the study were more accessible to them. It might also be that this age group is more exposed to ticks, as suggested by the peak in Lyme disease incidence reported amongst the $60-75$ years old age group in southern Sweden (Berglund et al., 1995). The TBEV vaccination coverage for the Swedish participants in our study was higher than the overall vaccination coverage of the Swedish population (Kunze, 2007), suggesting the participants in our study have a higher awareness of TBDs, that they visit TBE endemic areas more frequently, or are generally more anxious about TBDs. The fact that as much as 18 participants reported having had TBE previously, points to an overrepresentation of this group in willingness to take part in our TBD STING study. This possible population bias suggests that the results obtained in this study might not be representative for the whole population in risk of acquiring tick-bites.

In conclusion, we found a low prevalence of TBEV in ticks detached from humans, and accordingly, an overall low risk for transmission of TBEV. Because of few infected ticks, no certain conclusions could be drawn regarding the risk of clinical outcome in relation to 
duration of blood meal and virus copy number in the tick, but our results suggests an increasing virus amount in the ticks with longer feeding-time, possibly having clinical implications. One out of two unvaccinated persons, bitten by TBEV infected ticks, got a plausible TBEV viremia. We show that antibody responses may occur without clinical symptoms, and also that a bite by a TBEV infected tick with a low virus amount and short feeding duration not always leads to an antibody response or clinical symptoms. This study could not answer what the risk of CNS-involvement is when infected by TBEV.

\section{Acknowledgements}

We thank the staff at the PHCs involved in the TBD STING study for all their valuable work. The additional members of the TBD STING study group, consisting of Clas Ahlm, Johan Berglund, Anna J Henningsson, Peter Nolskog, Katharina Ornstein, Sten-Anders Carlsson, Christina Ekerfelt, and specially Liselott Lindvall and Mari-Anne Åkeson, that handled the core study logistics, and Susanne Olausson handling all the participants on the Åland Islands. We would like to thank Novartis vaccines (previously Chiron) for the K23 TBEV-vaccine antigen. We would also like to thank Andrew Jenkins for valuable contribution with editing the manuscript.

This study was supported by grants from The Swedish Research Council branch of Medicine, The Medical Research Council of South-East Sweden, Grants from the County Council of Östergötland, the Wilhelm and Else Stockmann Foundation and the Foundation for Åland Medical Research of the Åland Culture Foundation.

\section{References}


Alekseev, A.N., Burenkova, L.A., Vasilieva, I.S., Dubinina, H.V., Chunikhin, S.P., 1996. Preliminary studies on virus and spirochete accumulation in the cement plug of ixodid ticks. Exp. Appl. Acarol. 20, 713-723.

Alekseev, A.N., Dubinina, H.V., Jääskeläinen, A.E., Vapalahti, O., Vaheri, A., 2007. First report on tick-borne pathogens and exoskeletal anomalies in Ixodes persulcatus schulze (Acari: Ixodidae) collected in Kokkola coastal region, Finland. Int. J. Acarol. $33,253-258$.

Andreassen, A., Jore, S., Cuber, P., Dudman, S., Tengs, T., Isaksen, K., Hygen, H.O., Viljugrein, H., Anestad, G., Ottesen, P., Vainio, K., 2012. Prevalence of tick borne encephalitis virus in tick nymphs in relation to climatic factors on the southern coast of Norway. Parasit. Vectors 5, 177.

Belova, O.A., Burenkova, L.A., Karganova, G.G., 2012. Different tick-borne encephalitis virus (TBEV) prevalences in unfed versus partially engorged ixodid ticks - Evidence of virus replication and changes in tick behavior. Ticks Tick-Borne Dis. 3, 240-246.

Berglund, J., Eitrem, R., Ornstein, K., Lindberg, A., Ringnér, Å., Elmrud, H., Carlsson, M., Runehagen, A., Svanborg, C., Norrby, R., 1995. An Epidemiologic Study of Lyme Disease in Southern Sweden. N. Engl. J. Med. 333, 1319-1324.

Bormane, A., Lucenko, I., Duks, A., Mavtchoutko, V., Ranka, R., Salmina, K., Baumanis, V., 2004. Vectors of tick-borne diseases and epidemiological situation in Latvia in 19932002. Int. J. Med. Microbiol. 293 Suppl 37, 36-47.

Brinkley, Nolskog, P., Golovljova, I., Lundkvist, A., Bergstrom, T., 2008. Tick-borne encephalitis virus natural foci emerge in western Sweden. Int. J. Med. Microbiol. 298, 73-80.

Crippa, M., Rais, O., Gern, L., 2002. Investigations on the mode and dynamics of transmission and infectivity of Borrelia burgdorferi sensu stricto and Borrelia afzelii in Ixodes ricinus ticks. Vector Borne Zoonotic Dis. 2, 3-9.

Ecker, M., Allison, S.L., Meixner, T., Heinz, F.X., 1999. Sequence analysis and genetic classification of tick-borne encephalitis viruses from Europe and Asia. J. Gen. Virol. 80 ( Pt 1), 179-185.

Elväng, A., Melik, W., Bertrand, Y., Lönn, M., Johansson, M., 2011. Sequencing of a tickborne encephalitis virus from Ixodes ricinus reveals a thermosensitive RNA switch significant for virus propagation in ectothermic arthropods. Vector Borne Zoonotic Dis. 11, 649-658.

Golovljova, I., Vene, S., Sjölander, K.B., Vasilenko, V., Plyusnin, A., Lundkvist, A., 2004. Characterization of tick-borne encephalitis virus from Estonia. J. Med. Virol. 74, 580588.

Gray, J., Stanek, G., Kundi, M., Kocianova, E., 2005. Dimensions of engorging Ixodes ricinus as a measure of feeding duration. Int. J. Med. Microbiol. 295, 567-572.

Günther, G., Haglund, M., Lindquist, L., Forsgren, M., Sköldenberg, B., 1997. Tick-bone encephalitis in Sweden in relation to aseptic meningo-encephalitis of other etiology: a prospective study of clinical course and outcome. J. Neurol. 244, 230-238.

Gäumann, R., Mühlemann, K., Strasser, M., Beuret, C.M., 2010. High-throughput procedure for tick surveys of tick-borne encephalitis virus and its application in a national surveillance study in Switzerland. Appl. Environ. Microbiol. 76, 4241-4249.

Haglund, M., Forsgren, M., Lindh, G., Lindquist, L., 1996. A 10-year follow-up study of tickborne encephalitis in the Stockholm area and a review of the literature: need for a vaccination strategy. Scand. J. Infect. Dis. 28, 217-224.

Haglund, M., Günther, G., 2003. Tick-borne encephalitis--pathogenesis, clinical course and long-term follow-up. Vaccine 21 Suppl 1, S11-18. 
Haglund, M., Vene, S., Forsgren, M., Günther, G., Johansson, B., Niedrig, M., Plyusnin, A., Lindquist, L., Lundkvist, A., 2003. Characterisation of human tick-borne encephalitis virus from Sweden. J. Med. Virol. 71, 610-621.

Han, X., Juceviciene, A., Uzcategui, N.Y., Brummer-Korvenkontio, H., Zygutiene, M., Jääskeläinen, A., Leinikki, P., Vapalahti, O., 2005. Molecular epidemiology of tickborne encephalitis virus in Ixodes ricinus ticks in Lithuania. J. Med. Virol. 77, 249256.

Jaenson, T., Hjertquist, M., Lundkvist, Å., 2012. År 2011 toppar TBE-incidensen. Lakartidningen 109, 343-346.

Jääskeläinen, A., Korhonen, T., Vapalahti, O., 2011. Tick-borne encephalitis in Finland. EpiNorth 40-43.

Jääskeläinen, A.E., Sironen, T., Murueva, G.B., Subbotina, N., Alekseev, A.N., Castren, J., Alitalo, I., Vaheri, A., Vapalahti, O., 2010. Tick-borne encephalitis virus in ticks in Finland, Russian Karelia and Buryatia. J. Gen. Virol. 91, 2706-2712.

Jääskeläinen, A.E., Tikkakoski, T., Uzcátegui, N.Y., Alekseev, A.N., Vaheri, A., Vapalahti, O., 2006. Siberian subtype tickborne encephalitis virus, Finland. Emerg. Infect. Dis. $12,1568-1571$.

Kahl, O., Janetzki-Mittmann, C., Gray, J.S., Jonas, R., Stein, J., de Boer, R., 1998. Risk of infection with Borrelia burgdorferi sensu lato for a host in relation to the duration of nymphal Ixodes ricinus feeding and the method of tick removal. Int. J. Med. Microbiol. 287, 41-52.

Kaiser, R., 1999. The clinical and epidemiological profile of tick-borne encephalitis in southern Germany 1994-98 A prospective study of 656 patients. Brain 122, 20672078.

Kaiser, R., 2011. [Long-term prognosis of patients with primary myelitic manifestation of tick-borne encephalitis: a trend analysis covering 10 years]. Nervenarzt 82, 10201025 .

Klaus, C., Hoffmann, B., Hering, U., Mielke, B., Sachse, K., Beer, M., Süss, J., 2010. Tickborne encephalitis (TBE) virus prevalence and virus genome characterization in fieldcollected ticks (Ixodes ricinus) from risk, non-risk and former risk areas of TBE, and in ticks removed from humans in Germany. Clin. Microbiol. Infect. 16, 238-244.

Korenberg, E.I., Gorban, L.Y., Kovalevskii, Y.V., Frizen, V.I., Karavanov, A.S., 2001. Risk for human tick-borne encephalitis, borrelioses, and double infection in the pre-Ural region of Russia. Emerg. Infect. Dis. 7, 459-462.

Korenberg, E.I., Kovalevskii YuV, 1995. Variation in parameters affecting risk of human disease due to TBE virus. Folia Parasitol. (Praha) 42, 307-312.

Kotovich, L., 2012. Tick-Borne Encephalitis in the republic of Karelia, Russia. EpiNorth 6061.

Kunze, U., 2007. Tick-borne encephalitis: from epidemiology to vaccination recommendations in 2007 New issues - best practices. Wien. Med. Wochenschr. 157, $228-232$.

Larkin, M.A., Blackshields, G., Brown, N.P., Chenna, R., McGettigan, P.A., McWilliam, H., Valentin, F., Wallace, I.M., Wilm, A., Lopez, R., Thompson, J.D., Gibson, T.J., Higgins, D.G., 2007. Clustal W and Clustal X version 2.0. Bioinforma. Oxf. Engl. 23, 2947-2948.

Melik, W., Nilsson, A.S., Johansson, M., 2007. Detection strategies of tick-borne encephalitis virus in Swedish Ixodes ricinus reveal evolutionary characteristics of emerging tickborne flaviviruses. Arch. Virol. 152, 1027-1034. 
Mickiene, A., Laiskonis, A., Günther, G., Vene, S., Lundkvist, A., Lindquist, L., 2002. Tickborne encephalitis in an area of high endemicity in lithuania: disease severity and long-term prognosis. Clin. Infect. Dis. 35, 650-658.

Mickiené, A., Vene, S., Golovljova, I., Laiskonis, A., Lindquist, L., Plyusnin, A., Lundkvist, A., 2001. Tick-borne encephalitis virus in Lithuania. Eur. J. Clin. Microbiol. Infect. Dis. 20, 886-888.

Schwaiger, M., Cassinotti, P., 2003. Development of a quantitative real-time RT-PCR assay with internal control for the laboratory detection of tick borne encephalitis virus (TBEV) RNA. J. Clin. Virol. 27, 136-145.

Senigl, F., Grubhoffer, L., Kopecky, J., 2006. Differences in maturation of tick-borne encephalitis virus in mammalian and tick cell line. Intervirology 49, 239-248.

Süss, J., 2011. Tick-borne encephalitis 2010: Epidemiology, risk areas, and virus strains in Europe and Asia—An overview. Ticks Tick-Borne Dis. 2, 2-15.

Süss, J., Klaus, C., Diller, R., Schrader, C., Wohanka, N., Abel, U., 2006. TBE incidence versus virus prevalence and increased prevalence of the TBE virus in Ixodes ricinus removed from humans. Int. J. Med. Microbiol. 296 Suppl 40, 63-68.

Süss, J., Schrader, C., Falk, U., Wohanka, N., 2004. Tick-borne encephalitis (TBE) in Germany--epidemiological data, development of risk areas and virus prevalence in field-collected ticks and in ticks removed from humans. Int. J. Med. Microbiol. 293 Suppl 37, 69-79.

Tamura, K., Peterson, D., Peterson, N., Stecher, G., Nei, M., Kumar, S., 2011. MEGA5: molecular evolutionary genetics analysis using maximum likelihood, evolutionary distance, and maximum parsimony methods. Mol. Biol. Evol. 28, 2731-2739.

Tonteri, E., Jääskeläinen, A.E., Tikkakoski, T., Voutilainen, L., Niemimaa, J., Henttonen, H., Vaheri, A., Vapalahti, O., 2011. Tick-borne encephalitis virus in wild rodents in winter, Finland, 2008-2009. Emerg. Infect. Dis. 17, 72-75.

Vene, S., Haglund, M., Vapalahti, O., Lundkvist, A., 1998. A rapid fluorescent focus inhibition test for detection of neutralizing antibodies to tick-borne encephalitis virus. J. Virol. Methods 73, 71-75.

Wilhelmsson, P., Fryland, L., Börjesson, S., Nordgren, J., Bergström, S., Ernerudh, J., Forsberg, P., Lindgren, P.-E., 2010. Prevalence and diversity of Borrelia species in ticks that have bitten humans in Sweden. J. Clin. Microbiol. 48, 4169-4176. 


\section{Legends to illustrations}

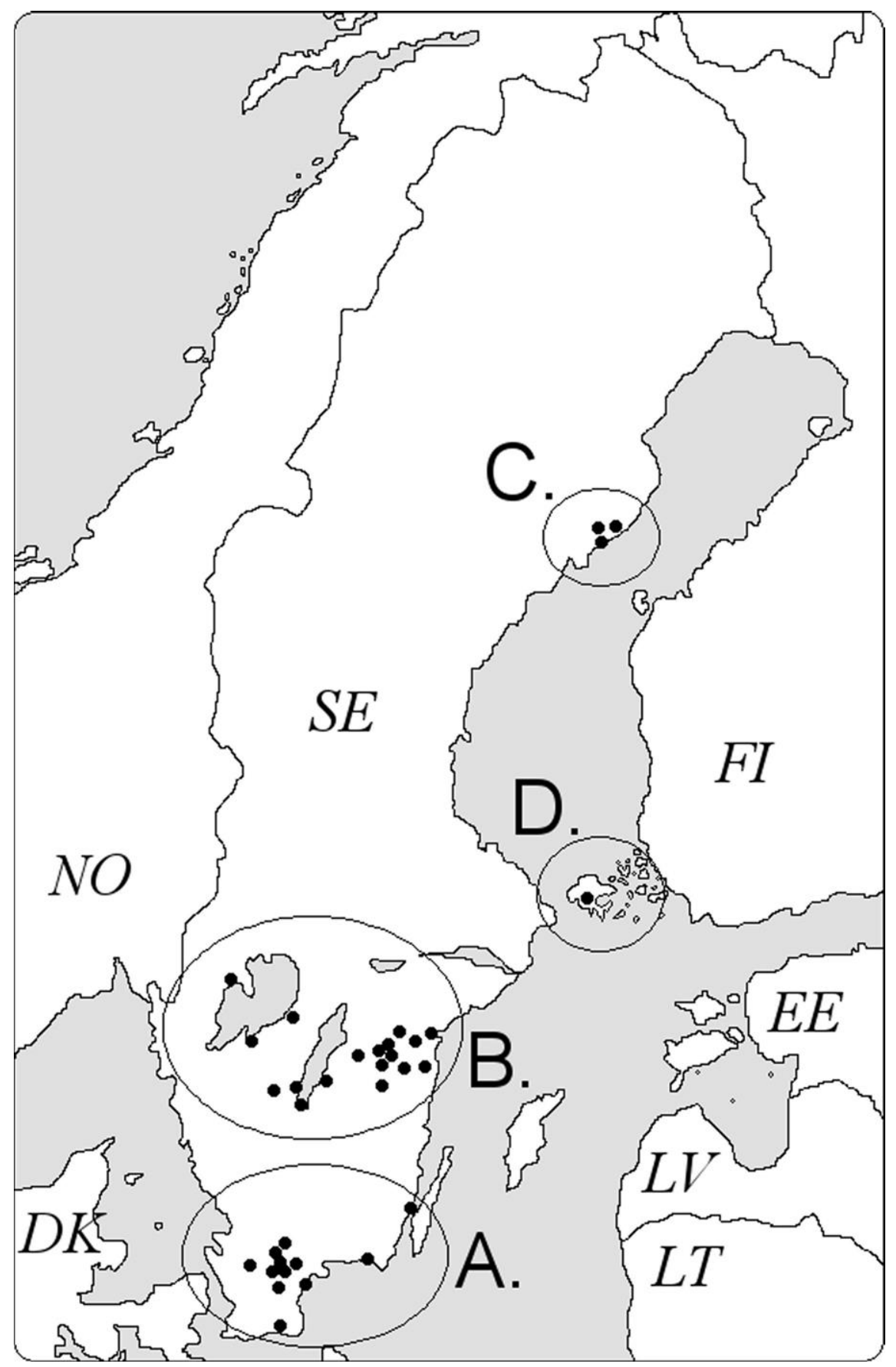

Figure 1. Location of the 34 primary health care centers (PHCs), from where tick-bitten persons were recruited, divided into four geographical regions. A: Southernmost Sweden (10 PHCs), B: South Central Sweden (20 PHCs), C: Northern Sweden (3 PHCs), and D: the Åland Islands (1 PHC). SE: Sweden, NO:Norway, FI: Finland, DK: Denmark, EE: Estonia, LV: Latvia, LT: Lithuania. 


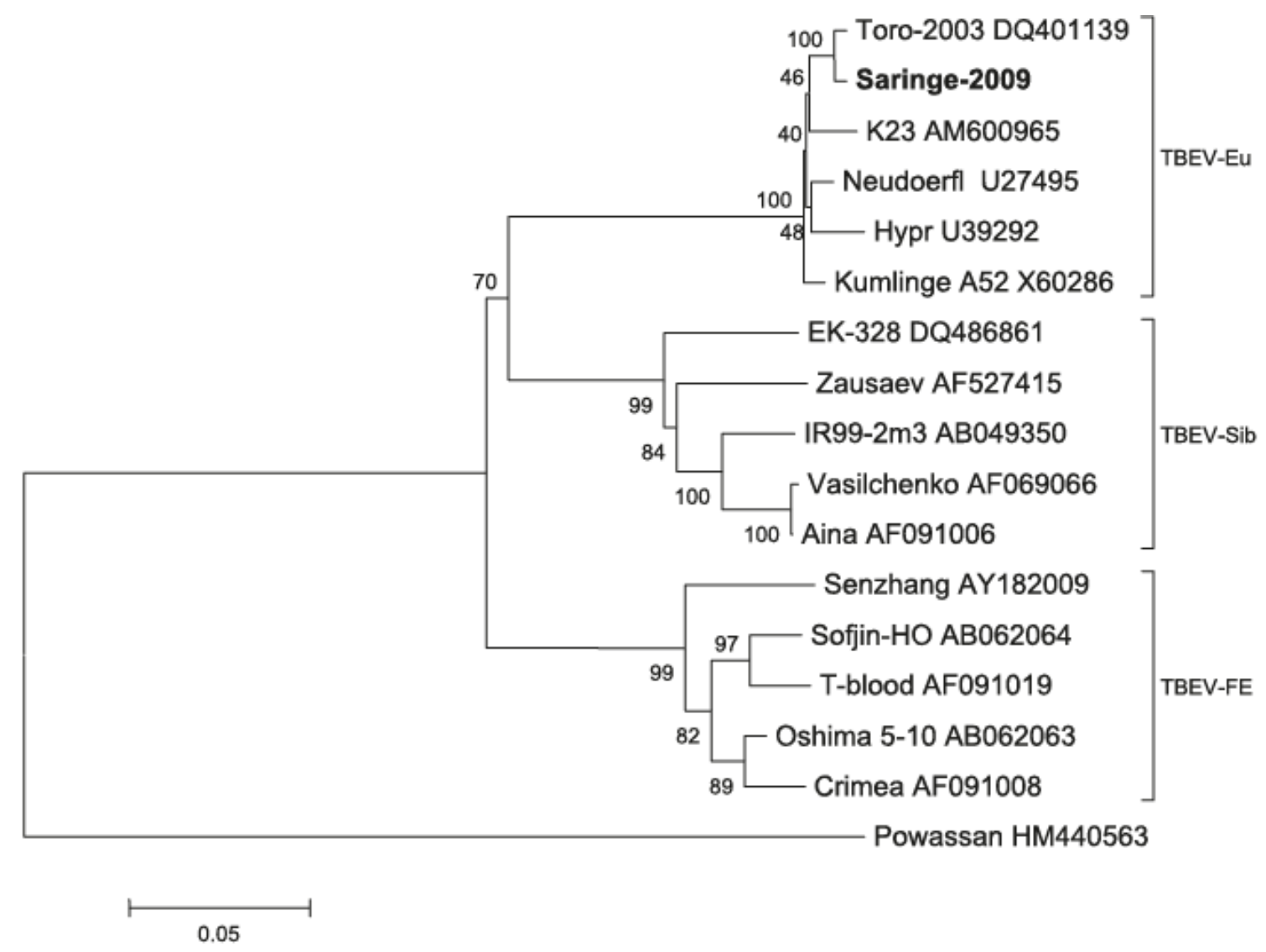

Figure 2. Neighbour-Joining phylogenetic analysis of the E-gene (1,488 bp) of TBEV strain Saringe-2009. Inferred from 1,000 bootstrap replicates in MEGA5 (Tamura et al., 2011). The tree is rooted by the Powassan virus and drawn to scale, with branch lengths corresponding to substitutions per site. The TBEV strains are grouped into 3 subtypes: The European (TBEVEu), The Siberian (TBEV-Sib) and the Far-Eastern (TBEV-FE). 